\title{
白唇竹叶青蛇毒 5'-核苷酸酶的分离纯化及性质
}

\author{
陈 夏，余晓东"，邓 敏，李 卉，林亦心，和七一，柳建平 \\ （重庆师范大学 生命科学学院, 重庆市生物活性物质工程研究中心, 重庆市动物生物学重点实验室, 重庆 400047)
}

\begin{abstract}
摘要: 用 DEAE-SephadexA-25、Sephadex-G-100 和 CM-SephadexC-50 三步柱层析分离法, 从白唇竹叶青 (Trimeresurus albolabris) 蛇毒中分离纯化出具有 5'-核苷酸酶活性的组分。SDS-聚丙烯酰胺凝胶电泳测定其分子 量为 $48.03 \mathrm{kDa}, \mathrm{HPLC}$ 柱层析图谱为单一峰。该组分是一个糖蛋白, 以一磷酸腺苷 (AMP) 为底物时, 其酶活力 为 $330.33 \mu \mathrm{g} \mathrm{Pi} /(\mathrm{min} \cdot \mathrm{mg})$; 而以二磷酸腺苷 ( $\mathrm{ADP}$ ) 为底物时, 其酶活力为 $123.56 \mu \mathrm{g} \mathrm{Pi} /(\mathrm{min} \cdot \mathrm{mg})$ 。金属 离子 $\mathrm{Zn}^{2+} 、 \mathrm{Fe}^{3+}$ 和 $\mathrm{Cu}^{2+}$ 对 $5^{\prime}$-核苷酸酶活性有显著的抑制作用, EDTA 可完全抑制其酶活性。该酶的最适 $\mathrm{pH}$ 为 9 , 最适温度为 $50^{\circ} \mathrm{C}$ 。该组分还具有抑制由 $\mathrm{ADP}$ 诱导的血小板聚集的生物功能。
\end{abstract}

关键词: 白唇竹叶青蛇毒; 5'-核苷酸酶; 分离纯化

中图分类号: Q502; Q959.6 文献标识码：A 文章编号：0254-5853-(2008)04-0399-06

\section{Purification and Characterization of 5'-nucleotidase from Trimeresurus albolabris Venom}

\author{
CHEN Xia, YU Xiao-dong*, DENG Min, LI Hui, LIN Yi-xin, HE Qi-yi, LIU Jian-ping \\ (College of Life Science, Chongqing Normal University; Chongqing Engineering Research Center of Bioactive Substance; \\ Chongqing Key Laboratory of Animal Biology, Chongqing 400047)
}

\begin{abstract}
A 5'-nucleotidase was isolated and purified from the snake venom of T. albolabris using three steps of chromatography including DEAE-SephadexA-25, Sephadex-G-100 and CM-Sephadex C-50. Using SDS-PAGE and HPLC column chromatography the purified 5 '-nucleotidase proved to be homogenous. It was a glycoprotein with a molecular weight of $48.03 \mathrm{kDa}$. The enzymatic activities of the purified $5^{\prime}$-nucleotidase were $330.33 \mu \mathrm{g} \mathrm{Pi} / \mathrm{min} \mathrm{mg}$ and $123.56 \mu \mathrm{g} \mathrm{Pi} / \mathrm{min} \mathrm{mg}$ when using AMP (adenosine monophosphate) and ADP (adenosine diphosphate) as substrates, respectively. Metal ions, including $\mathrm{Zn}^{2+}, \mathrm{Fe}^{3+}$ and $\mathrm{Cu}^{2+}$, could inhibit $5^{\prime}$-nucleotidase activity, as did EDTA. Its optimum $\mathrm{pH}$ was nine and its optimum temperature was $50^{\circ} \mathrm{C}$. It has a potent inhibitory effect on rabbit platelet aggregation induced by ADP.
\end{abstract}

Key words: Trimeresurus albolabris; Snake venom; 5'-nucleotidase; Isolation and purification

5'-核苷酸酶是一种磷酸酯酶, 主要分布于动植 物细胞、细菌及动物毒液中 ( $\mathrm{Li}, 2004)$ 。它能水解 5 '-单核苷酸, 产生核苷。为此, 作为一种工具酶, 已被广泛用于基因工程和核酸研究。同时, 该酶也 具有重要的生物功能, 如在细胞生长发育、运动、 纤维蛋白合成、神经传递、提高表皮或内皮屏障功 能及淋巴细胞的黏附、再循环、免疫应答等方面均 发挥重要的作用（Colgan et al，2006）。不同生物 来源和同种生物不同组织细胞来源的 5'-核苷酸酶 的理化、酶学性质和生物功能存在一定的差异 (Sträter，2006 )。尤其是从不同种蛇毒来源的 5'-
核苷酸酶在生物功能上显示出差异, 如从棕点竹叶 青 (Trimeresurus. gramineus) 蛇毒中分离出的5'-核 苷酸酶，具有抑制由ADP、花生四烯酸（AA）、胶 原蛋白、低浓度凝血酶和Ionophor（A-23187）诱导 的血小板聚集的活性 (Ouyang \& Huang，1983); 从竹叶青(T. Stejnegeri)蛇毒分离出的 $5^{\prime}$-核苷酸酶能 抑制ADP、AA、TMVA、凝血酶诱导的血小板聚集， 而且对 ADP诱导的血小板聚集还有明显的解聚作 用 (Yu et al， 1997); 眼镜蛇（Naja naja）毒的5'核苷酸酶具有抗凝血作用 (Dhananiaya et al, 2006)。 我们曾对我国重庆金佛山产的白唇竹叶青 ( $T$.

收稿日期: 2008-01-30; 接受日期: 2008-05-27

基金项目：重庆市自然科学基金重点项目（CSTC2006BA5032)；重庆市教委基金项目(KJ080824)

*通讯作者 (Corresponding author), E-mail: yxd@cqnu.edu.cn 
albolabris）蛇毒粗毒进行检测，发现具有 $5^{\prime}$-核苷酸 酶活性, 但目前对该蛇毒 $5^{\prime}$-核苷酸酶的研究还未曾 见报道。为此, 本文从白唇竹叶青 (T. albolabris) 蛇毒粗毒中分离纯化该酶, 并对其理化和酶学性质 及对家兔血小板聚集的作用进行研究, 现将我们的 研究结果报道如下。

\section{1 材料与方法}

\section{1 材 料}

白唇竹叶青毒蛇采自重庆金佛山; 健康家兔, 体重 $5 \mathrm{~kg} \pm 20 \mathrm{~g}$, 购于重庆腾金生生物技术有限公司; DEAE-SephadexA-25 、CM-SephadexC-50 和 Sephadex G-100(superfine)购自 Amresham 公司; AMP 和 ADP 购自 Amresco 公司; 蛋白质 Markers 购自 Takara 公司; EDTA 和 PMSF 为 Sigma 公司产 品; 其他所用试剂均为国产分析纯。

\section{2 仪 器}

Himac CR 22E 高速冷冻离心机(日本, Hitachi), Modul YOD-230 真空冷冻干燥机(美国, Thermo savant), PB-10 酸度计(德国, 赛多利斯), Biologic Duoflow 层析系统和 UNIVERSAL HOOD II S.N 凝 胶成相系统(美国, Bio-rad), 高效液相色谱仪(HPLC) Waters600 (美国, Waters), 8500 型紫外分光光度计 (上海天美), LBY-NJ4 血小板聚集仪 (北京普利 生)。

\section{3 白唇竹叶青蛇毒 $5^{\prime}$-核苷酸酶的分离纯化}

白唇竹叶青蛇毒 $0.5 \mathrm{~g}$ 溶于 $0.05 \mathrm{~mol} / \mathrm{L}$ Tris-HCl缓 冲液 $3 \mathrm{~mL}(\mathrm{pH} 8.3)$ 中, $4^{\circ} \mathrm{C}$ 孵育过夜, 完全溶解后离 心 $10 \mathrm{~min}\left(4^{\circ} \mathrm{C}, 5000 \mathrm{r} / \mathrm{min}\right)$, 取上清, 上样于用同样 缓冲液平衡好的DEAE-SephadexA-25柱, 用 $0-1$ $\mathrm{mol} / \mathrm{L} \mathrm{NaCl}$ 溶液线性梯度洗脱; 将具有 $5^{\prime}$-核苷酸酶 活性强的分离峰收集浓缩后, 上样于 Sephadex G-100柱, 用 $0.01 \mathrm{~mol} / \mathrm{L}$ Tris-HCl缓冲液（pH7.6）洗 脱; 再将得到的具有 $5^{\prime}$-核苷酸酶活性强的分离峰收 集浓缩后, 上样于 CM-SephadexC-50柱, 用 0.01 $\mathrm{mol} / \mathrm{L}$ 醋酸钠缓冲液 $(\mathrm{pH} 5.8)$ 洗脱, 并用 $0-1 \mathrm{~mol} / \mathrm{L}$ $\mathrm{NaCl}$ 溶液线性梯度洗脱。每次收集的活性组分进行 Sephadex G-25除盐、浓缩, 然后上下一步色谱柱。

\section{4 纯度鉴定和分子量的测定}

SDS-聚丙烯酰胺凝胶电泳法测定分子量：分离 胶浓度 $15 \%$, 浓缩胶浓度 $5 \%$, 电泳完后用考马斯亮 蓝R250染色, 用含 $25 \%$ 乙醇和 $10 \%$ 冰醋酸的混合液 脱色。
HPLC柱层析: 取最后得到的样品 $20 \mathrm{mg}$, 溶于 $750 \mu \mathrm{L} 0.1 \% \mathrm{TFA}$ 溶液, $4500 \mathrm{r} / \mathrm{min}$ 离心 $5 \mathrm{~min}$, 取上清 液, 上样于用 $0.1 \% \mathrm{TFA}$ 溶液平衡过的 $\mathrm{C}_{18}$ 柱, 用 $0-$ $66.5 \%$ 乙腈(溶于 $0.1 \% \mathrm{TFA}$ 溶液)梯度洗脱, 最后用 $66.5 \%$ 乙腈 $0.1 \% \mathrm{TFA}$ 溶液洗脱。

\section{5 糖蛋白测定}

参照 Guang \& Qi（1982）方法进行。

\subsection{5 '-核苷酸酶、ADP 酶活力测定}

参照 The Fourth Section of Yunnan Institute of Zooloogy (1976)方法进行, 按 Jesudium et al (1976) 方法测定无机磷的生成。

\section{7 金属离子对酶活力的影响}

各种金属离子盐 $\left(\mathrm{Ca}^{2+} 、 \mathrm{Mg}^{2+} 、 \mathrm{Zn}^{2+} 、 \mathrm{Cu}^{2+}\right.$ 和 $\mathrm{Fe}^{3+}$ ) 配成 $5 \mathrm{mmol} / \mathrm{L}$, 与等体积酶溶液 $(300 \mu \mathrm{g} / \mathrm{mL})$ 混合, $37^{\circ} \mathrm{C}$ 保温 $1 \mathrm{~h}$ 后稀释成 $50 \mu \mathrm{g} / \mathrm{mL}$, 然后测定 其 5'-核苷酸酶活性(以 AMP 为底物)。EDTA 作用 于酶后, 再加入过量金属离子, $37^{\circ} \mathrm{C}$ 保温 $1 \mathrm{~h}$, 检测 5'-核苷酸酶活性。以不加任何离子的一组作为对照 组, 酶活力设为 $100 \%$ 。

\section{8 抑制剂对酶活性的影响}

4 种化学抑制剂（苯甲脒、 $\beta$-颈基乙醇、PMSF 和 EDTA）分别配成 $5 \mathrm{mmol} / \mathrm{L}$ 溶液, 分别与等体积 酶溶液 $(300 \mu \mathrm{g} / \mathrm{mL})$ 混合, $37^{\circ} \mathrm{C}$ 保温 $1 \mathrm{~h}$ 后稀释成 $50 \mu \mathrm{g} / \mathrm{mL}$, 然后测定 $5^{\prime}$ - 核苷酸酶活性(底物为 AMP)。 以不加任何试剂的一组作为对照组, 酶活力设为 $100 \%$ 。

\section{$1.9 \mathrm{pH}$ 对酶活性的影响}

不同 $\mathrm{pH}$ 缓冲液体系组成如下: $20 \mathrm{mmol} / \mathrm{L}$ 醋酸 -醋酸钠缓冲液 ( $\mathrm{pH} 4.0-5.0) ; 20 \mathrm{mmol} / \mathrm{L}$ 磷酸二氢钾 -氢氧化钠缓冲液 $(\mathrm{pH} 6.0-7.0) ; 50 \mathrm{mmol} / \mathrm{L}$ Tris-盐酸 缓冲液 $(\mathrm{pH} 8.0-9.0) ; 50 \mathrm{mmol} / \mathrm{L}$ 碳酸氢钠-氢氧化钠 缓冲液 $(\mathrm{pH} 10.0-11.0)$, 所有缓冲液均含有 0.5 $\mathrm{mmol} / \mathrm{L} \mathrm{CaCl}{ }_{2}$ 和 $10 \mathrm{mmol} / \mathrm{L} \mathrm{NaCl}$ 以维持相同盐浓 度。样品分别与上述缓冲液混合, 室温作用 $1 \mathrm{~h}$, 再 测定其 $5^{\prime}$-核苷酸酶活力(AMP 为底物)。以最高酶活 力定义为 $100 \%$, 计算其他不同 $\mathrm{pH}$ 条件下的相对酶 活力, 以 $\mathrm{pH}$ 与酶的相对活力作图。

\subsection{0 温度对酶活性的影响}

样品置不同温度 $(10 、 20 、 30 、 40 、 50 、 60 、 70$ 和 $80^{\circ} \mathrm{C}$ )下保温 $1 \mathrm{~h}$, 然后在同样温度下测定其 $5^{\prime}$ 核苷酸酶活性(AMP 为底物)。以最高酶活力定义为 $100 \%$, 计算其他不同温度条件下的相对酶活力, 以 温度与酶的相对活力作图。 


\subsection{1 血小板聚集实验}

富血小板血浆的制备：新鲜的兔血收集于硅烷 化的塑料管内, 并以 $3.8 \%$ 柠檬酸钠 $9: 1(\mathrm{~V} / \mathrm{V})$ 抗 凝，室温离心 $10 \mathrm{~min}(1000 \mathrm{r} / \mathrm{min})$ ，取上层液即为 富血小板血浆 (PRP)。剩余血液继续离心 $15 \min$ (3 $000 \mathrm{r} / \mathrm{min}$ ), 分离出贫血小板血浆 (PPP), 作为测定 时的对照或调节 PRP 中的血小板数。

血小板聚集性测定：按 Born 氏比浊法原理，采 用 LBY-NJ4 血小板聚集仪进行测定。测定结果按下 式计算:

（对照最大聚集百分率一
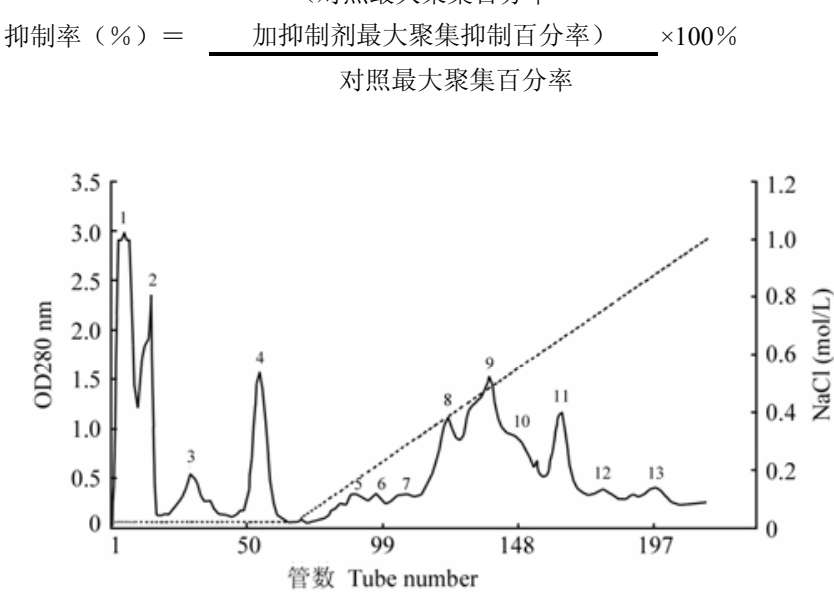

图 1 DEAE-SephadexA-25 柱色谱图

Fig. 1 DEAE-SephadexA-25 ion exchange chromatograms 层析柱: $2.0 \mathrm{~cm} \times 60 \mathrm{~cm}$; 流速: $24 \mathrm{~mL} / \mathrm{h}$; 每管收集体积: $4 \mathrm{~mL}$ 。

Column volume: $2.0 \mathrm{~cm} \times 60 \mathrm{~cm}$; flow rate: $24 \mathrm{~mL} / \mathrm{h}$; liquid volume of each tube: $4 \mathrm{~mL}$

核苷酸酶活性。将峰III合并收集后, 在Sephadex G-25上脱盐后进行检测。

\subsection{5 '-核苷酸酶的纯度}

纯化后的 5'-核苷酸酶组分作SDS-PAGE, 其结果 如图4所示, 呈现为单一条带。测得相对分子量约为 $48.03 \mathrm{kDa}$ 。

将 CM-SephadexC-50 层析后的第 III 蜂所得样 品上 HPLC 层析后, 其色谱结果如图 5 所示, 在 10 $-12 \mathrm{~min}$ 时洗脱下一个单一的蛋白峰。

\section{5 糖蛋白测定结果}

聚丙烯酰胺凝胶电泳后的条带切成两份, 分别 用考马斯亮兰和过碘酸-Schiff's 试剂染色, 条带呈 粉红色, 且位置与考马斯亮兰染色位置一致, 表明 它是糖蛋白。

\subsection{5 -核苷酸酶、ADP 酶活力测定结果}

以 AMP 为底物时 $5^{\prime}$-核苷酸酶活力为 $330.33 \mu \mathrm{g}$

\section{2 结 果}

\subsection{DEAE-SephadexA-25 柱色谱结果}

分离图谱如图1所示, 共分离出 13 个峰, 其中峰 I 的 5'-核苷酸酶活性最强, 将峰 I 收集并在 Sephadex G-25上脱盐浓缩后做进一步纯化。

\subsection{Sephadex G-100柱色谱结果}

分离图谱如图 2 所示, 共得 3 个峰, 其中峰 II 具 有5'-核苷酸酶活性。将峰 II 合并收集，浓缩后做进 一步纯化。

\subsection{CM-SephadexC-50 柱色谱结果}

分离图谱如图3所示, 共得 3 个峰, 其中峰III具 5'-

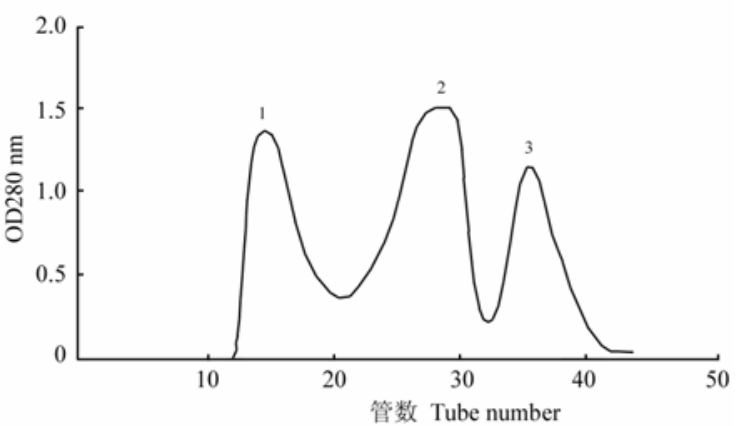

图 2 Sephadex G-100 柱色谱图

Fig. 2 Sephadex-G-100 gel filtration chromatograms 层析柱: $1.0 \mathrm{~cm} \times 100 \mathrm{~cm}$; 流速: $6 \mathrm{~mL} / \mathrm{min}$; 每管收集体积: $3 \mathrm{~mL}$ 。 Column volume: $1.0 \mathrm{~cm} \times 100 \mathrm{~cm}$; flow rate: $6 \mathrm{~mL} / \mathrm{min}$; liquid volume of each tube: $3 \mathrm{~mL}$.

$\mathrm{Pi} /(\mathrm{min} \cdot \mathrm{mg})$, 以 $\mathrm{ADP}$ 为底物时 $5^{\prime}$-核苷酸酶活力 为 $123.56 \mu \mathrm{g} \mathrm{Pi} /(\mathrm{min} \cdot \mathrm{mg})$ 。

\section{7 金属离子和抑制剂对酶活力的影响}

金属离子及抑制剂对 5'-核苷酸酶活力的影响 (AMP 为底物) 结果如表 1 所示, $\mathrm{Zn}^{2+} 、 \mathrm{Fe}^{3+}$ 和 $\mathrm{Cu}^{2+}$ 对 $5^{\prime}$-核苷酸酶活性有显著的抑制作用, EDTA 完全 抑制了该酶的活力。

\section{$2.8 \mathrm{pH}$ 对酶活性的影响}

$\mathrm{pH}$ 对白唇竹叶青蛇毒 $5^{\prime}$-核苷酸酶活性的影响 (AMP 为底物) 结果见图 6, 显示其该酶的最适 $\mathrm{pH}$ 为 9 。

\section{9 温度对酶活性的影响}

温度对白唇竹叶青蛇毒 $5^{\prime}$-核苷酸酶活性的影响 (AMP 为底物) 结果见图 7, 显示该酶的最适温度 为 $50^{\circ} \mathrm{C}$ 。

\subsection{0 血小板聚集测定结果}




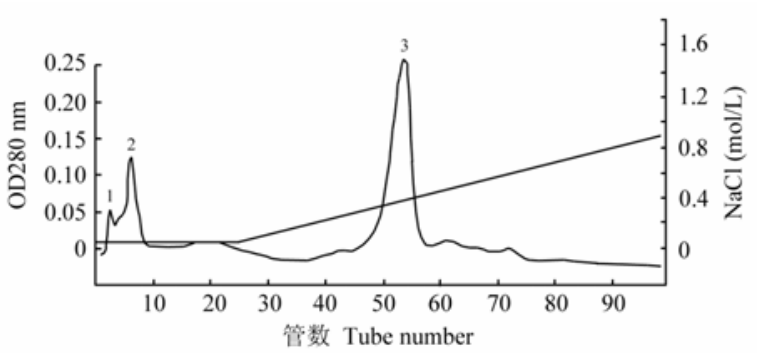

图 3 CM-SephadexC-50 柱色谱图

Fig. 3 CM-SephadexC-50 ion exchange chromatograms 层析柱: $2.0 \mathrm{~cm} \times 30 \mathrm{~cm}$; 流速: $20 \mathrm{~mL} / \mathrm{h}$; 每管收集体积: $3 \mathrm{~mL}$ 。 Column volume: $2.0 \mathrm{~cm} \times 30 \mathrm{~cm}$; flow rate: $20 \mathrm{~mL} / \mathrm{h}$; liquid volume of each tube: $3 \mathrm{~mL}$.

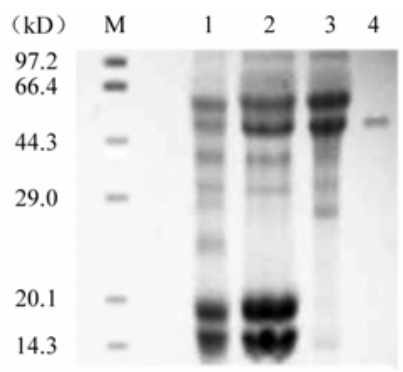

图 4 白唇竹叶青蛇毒 5'-核苷酸酶的 SDS-PAGE 图谱

Fig. 4 SDS-PAGE pattern of the 5 '-nucleotidase of snake venom (Trimeresurus albolabris)

泳道 M: 蛋白质 marker; 1: 粗毒样品; 2: DEAE-SephadexA-25 第 1 峰 样品; 3: Sephadex G-100 第 2 峰样品; 4: CM-SephadexC-50 第 3 峰样品。 样品上样量分别为 $40 、 40 、 40$ 和 $6 \mu \mathrm{g}$ 。

Lane M: protein marker; 1: crude venom; 2: the first peak after DEAE-SephadexA-25; 3: the second peak after Sephadex G-100; 4: the third peak after CM-SephadexC-50. Each sample is 40, 40, 40, $6 \mu \mathrm{g}$.

表 1 金属离子及抑制剂对白唇竹叶青蛇毒 5 -核苷酸酶活力

( $\mu \mathrm{g} \mathrm{Pi} / \mathrm{min} \mathrm{mg})$ 的影响 $(n=3$, mean $\pm \mathrm{SD})$

Tab. 1 Effect of metal ions and inhibitors on the activity of 5'-nucleotidase ( $\mu \mathrm{g} \mathrm{Pi} / \mathrm{min} \mathrm{mg}$ ) from snake venom (Trimeresurus albolabris)

\begin{tabular}{cccccccccc}
\hline \multirow{2}{*}{ 对照 } & \multicolumn{4}{c}{ 金属离子 $(5 \mathrm{mmol} / \mathrm{L})$} \\
\cline { 2 - 10 } & $\mathrm{Mg}^{2+}$ & $\mathrm{Ca}^{2+}$ & $\mathrm{Zn}^{2+}$ & $\mathrm{Fe}^{3+}$ & $\mathrm{Cu}^{2+}$ & $\mathrm{EDTA}$ & $\mathrm{PMSF}$ & $\beta$ 一疏基乙醇 & 苯甲㜆 \\
\hline 100 & $98.4 \pm 0.7$ & $106.8 \pm 2.3$ & $3.2 \pm 1.0^{*}$ & $37.6 \pm 1.0^{*}$ & $0^{*}$ & $0^{*}$ & $91.8 \pm 3.1$ & $113 \pm 2.8$ & $97 \pm 0.6$ \\
\hline
\end{tabular}

The values represent the mean $\pm \mathrm{SD}, n=3, * P<0.01$. Student- $t$ analysis.

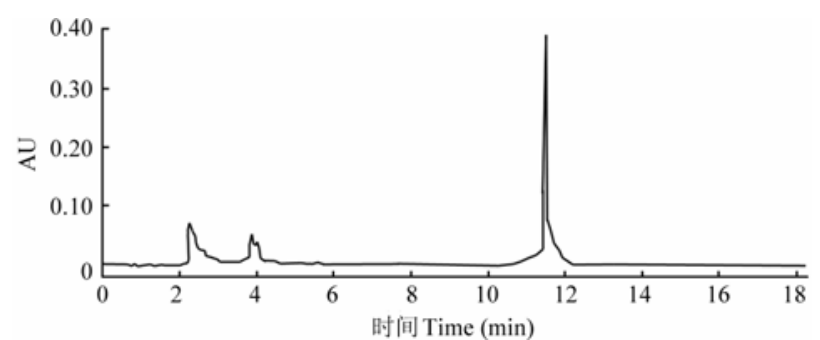

图 5 HPLC 柱层析色谱图

Fig. 5 HPLC chromatograms of 5'-nucleotidase 层析柱: 分析型 $\mathrm{C} 18$ 柱; 流速: $2 \mathrm{~mL} / \mathrm{min}$ 。 Column: $\mathrm{C} 18$ analysis; flow rate $: 2 \mathrm{~mL} / \mathrm{min}$.

白唇竹叶青蛇毒 5'-核苷酸酶对 ADP（20 $\mu \mathrm{mol} / \mathrm{L}$ ) 诱导的家兔血小板聚集具有明显的抑制作 用。抑制率与所用 $5^{\prime}$-核苷酸酶剂量相关(图 8)。

\section{3 讨 论}

通过 DEAE-SephadexA-25、Sephadex-G-100 和 CM-SephadexC-50 三步柱层析法, 我们从我国产的
白唇竹叶青蛇毒中首次分离纯化出了 5 -核苷酸酶 组分 (图 1, 2, 3), SDS-PAGE 和 HPLC 层析结果 皆提示该组分为单一组分(图 4,5), 其表观分子量 为 $48.03 \mathrm{kDa}$, 属糖蛋白。其理化和酶学性质实验表 明, 该 5'-核苷酸酶组分与竹叶青属的其他蛇毒来源 的 5'-核苷酸酶有较多相似点, 如它们皆为糖蛋白, 其酶活性能皆被 EDTA 抑制, $\mathrm{Zn}^{2+}$ 对其酶活性皆有 明显抑制作用; 也有不同处, 如 $\mathrm{Fe}^{3+}$ 离子不能完全 抑制白唇竹叶青蛇毒 $5^{\prime}$-核苷酸酶的活性, 而能完全 抑制竹叶青蛇毒 5'-核苷酸酶活性。在最适 $\mathrm{pH}$ 方面, 白唇竹叶青蛇毒的 $5^{\prime}$-核苷酸酶为 9, 与竹叶青蛇毒 中分离出的 5'-核苷酸酶相似 (Yu et al, 1996), 但 与从眼镜蛇(Najn naja atra)蛇毒中分离的 $5^{\prime}$-核苷酸 酶 (最适 $\mathrm{pH}$ 为 6.5-7.0) 和从蝮蛇(Agkistrodon blomhoffii)蛇毒中分离的 $5^{\prime}$-核苷酸酶 (最适 $\mathrm{pH}$ 为 6.8-6.9）不同。不同种属的蛇毒 $5^{\prime}$-核苷酸酶的这 些异同点可能与它们所处的分类种属亲缘关系有 


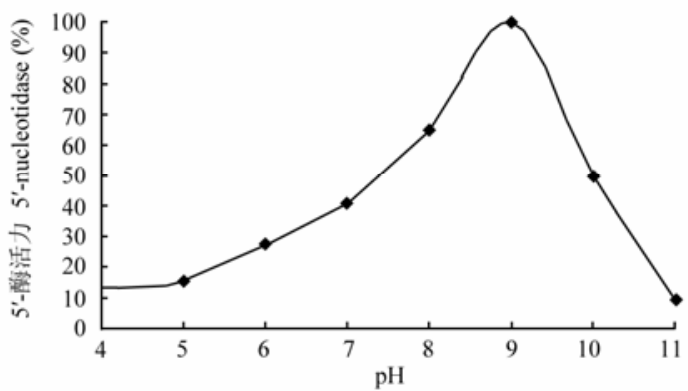

图 $6 \mathrm{pH}$ 对白唇竹叶青蛇毒 5'-核苷酸酶活力的影响 ( mean $\pm \mathrm{SD}, n=3$ )

Fig. 6 Effect of $\mathrm{pH}$ on the activity of 5 '-nucleotidase from snake venom (Trimeresurus albolabris) $\quad($ mean $\pm \mathrm{SD}, n=3)$

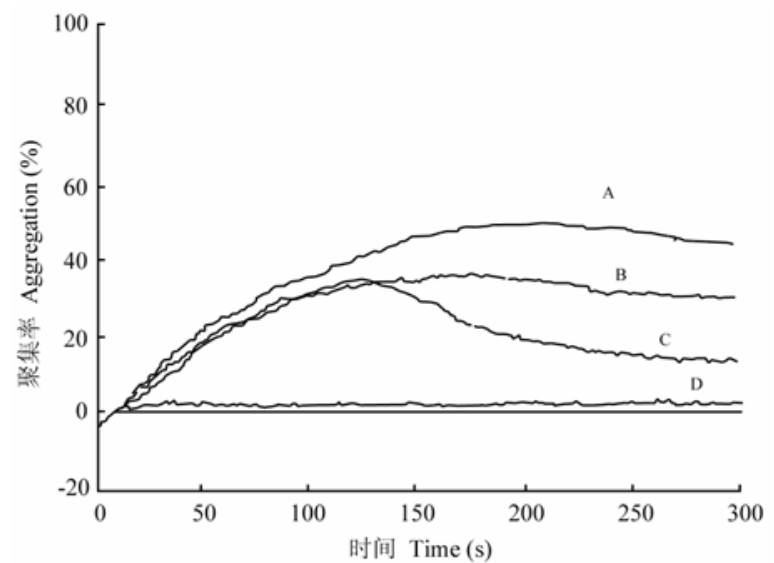

图 8 白唇竹叶青蛇毒 $5^{\prime}$-核苷酸酶对 ADP $(20 \mu \mathrm{mol} / \mathrm{L})$ 诱导的家兔血小板聚集的影响(mean $\pm \mathrm{SD}, n=3$ )

Fig. 8 The effect of T. albolabris 5 '-nucleotidase on rabbit platelet aggregation induced by ADP $(20 \mu \mathrm{mol} / \mathrm{L})(\operatorname{mean} \pm \mathrm{SD}, n=3)$

$\mathrm{A}$ ：对照，未加 5'-核苷酸酶； B、C、D：加 5'-核苷酸酶（分别为 25 , 50 和 $100 \mu \mathrm{g} / \mathrm{mL}$ )。

A: control, absence of 5'-nucleotidase; B, C and D: 5'-nucleotidase with respectively 25,50 and $100 \mu \mathrm{g} / \mathrm{mL}$

\section{参考文献:}

Chandrarajan J, Klein L. 1976. Determination of inorganic phosphorus in the presence of organic phosphorus and high concentrations of proteins [J]. Analytical Biochemistry,72(1-2) : 407-412.

Colgan SP, Eltzschig HK, Eckle T, Thompson LF. 2006. Physiological roles for ecto-5'-nucleotidase (CD73) [J]. Purinergic Signalling, 2: 351-360

Dhananjaya BL, Nataraju A, Rajesh R, Gowda CDR, Sharath BK,Vishwanath BS, D'Souza CJM. 2006. Anticoagulant effect of Naja naja venom 5'-nucleotidase: demonstration through the use of novel specific inhibitor, vanillic acid [J]. Toxicon, 48 (4): 411-421.

Guan LF, Qi ZW. 1982. Study on a thrombin-like enzyme from the venom of Agkistrodon Halyspallas- I.Purification and characterization of physicochemical and enzymatic properties [J]. Acta Biochinica et Biophysica Sinica, 14 (4) : 303-307. [管利丰, 戚正武. 1982. 蝮蛇

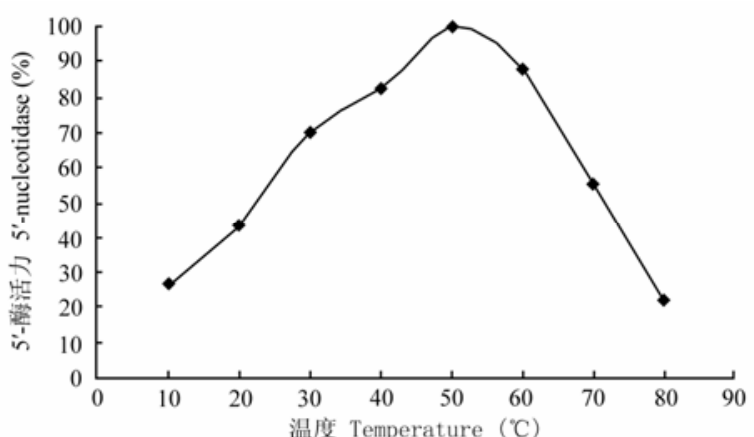

图 7 温度对白唇竹叶青蛇毒 5'-核苷酸酶活力的影响 ( $\operatorname{mean} \pm \mathrm{SD}, n=3$ )

Fig. 7 Effect of temperature on the activity of 5'-nucleotidase from snake venom (T. albolabris) $\quad($ mean $\pm \mathrm{SD}, n=3)$

关，值得进一步探讨。温度对白唇竹叶青蛇毒 5'核苷酸酶的影响实验表明, 最适温度在 $50^{\circ} \mathrm{C}$ 左右 (图 7), 并且在 $50^{\circ} \mathrm{C}$ 作用 $30 \mathrm{~min}$ 活性不变，提示 它是一个热稳定性蛋白。

蛇毒中的抗凝血因子很多, 直接作用于血小板 从而发挥促凝和抗凝血作用的组分也不少 (White, 2005; Oyama \& Takahashi，2007)。蛇毒5'-核苷酸 酶通过作用于血小板发挥抗凝血功能。Ouyang \& Huang (1983) 对棕点竹叶青蛇毒5'-核苷酸酶的研 究以及Yu et al (1997) 对竹叶青蛇毒5'-核苷酸酶的 研究结果表明, 蛇毒 5 '-核苷酸酶通过作用于血小板 发挥抗凝血功能。血小板聚集实验表明从白唇竹叶 青蛇毒得到的 $5^{\prime}$-核苷酸酶组分也能抑制由ADP诱 导的家兔血小板血浆的聚集，且抑制率与 $5^{\prime}$-核苷酸 酶的量呈正相关 (图8), 但其抗血小板聚集的详细 分子机制不清楚, 还有待于进一步研究。

(Ahallys Pallas)蛇毒类凝血酶的研究. I. 分离纯化及理化酶学性质的 鉴定. 生物化学与生物物理学报, 14 (4): 303-307.]

Li XB. 2004. The research progress of 5'-nucleotidase [J]. Foreign Medical Sciences Section of Pathophysiology and Clinical Medicine, 24 (2) : 122-124. [李晓波. 2004. 5'-核苷酸酶的研究进展. 国外医学·生理、病 理科学与临床分册, 24 (2)：122-124.]

Ouyang C, Huang TF. 1983. Inhibition of platelet aggregation by 5 '-nucleotidase purified from Trimeresurus gramineus snake venom [J]. Toxicon, 21 (4) : 491-501.

Oyama E, Takahashi H. 2007. Distribution of low molecular weight platelet aggregation inhibitors from snake venoms [J]. Toxicon, 49 (3): 293-298.

Sträter N. 2006. Ecto-5'-nucleotidase: Structure function relationships [J]. Purinergic Signalling , 2: 343-350. 
The Fourth Section Of Yunnan Zooloogy Institute. 1976. Studies on snake venoms and their utilization I . Estimation of enzymatic activities of the snake venoms in China[J]. Acta Biochinica et Biophysica Sinica, 8 (2): 157. [云南动物研究所第四室. 1976. 蛇毒的研究和利用. I. 我国常 见的蛇毒酶活力的测定. 生物化学与分子生物学学报, 8 (2): 157.]

White J. 2005. Snake venoms and coagulopathy [J]. Toxicon, 45 (8): 951-967.

Yu XD, Huang LN, Xiong YL. 1996. The study of Trimersesurus stejnegeri snake venom - the separat ion and purification of it's 5'-nucleotidase [J]. Journal of Chongqing Normal University (Natural Science Edition ),
13(4): 42-48. [余晓东, 黄立农, 熊郁良. 1996. 竹叶青(T rim eresu rus stejneg eri)蛇毒——对 5 '-核苷酸酶的分离纯化及理化和酶学性 质研究. 重庆师范大学学报 (自然科学版) , (4): 42-48.]

Yu XD, Huang LN, Xiong YL. 1997. The mechanism of 5'-nucleotidase purified from Trim ersesurus stejnegeri snake venom on platelet aggregation [J]. Journal of Chongqing Normal University (Natural Science Edition ), 14(2): 61-68. [余晓东, 黄立农, 熊郁良. 1997. 竹叶 青(Trimeresurus stejnegeri)蛇毒 5'-核苷酸酶对血小板聚集功能的抑 制机制研究. 重庆师范大学学报 (自然科学版), 14 (2): 61-68.] 